\title{
Hemodynamic study of unenhanced magnetic resonance angiography using spatial labeling with multiple inversion pulses sequence: a phantom study
}

\author{
Xiao Chen, Xiaoyan Meng, Di Zhu, Xianlun Zou, Yaqi Shen, Zhen Li, Jian Peng, Daoyu Hu \\ Department of Radiology, Tongji Hospital, Tongji Medical College, Huazhong University of Science and Technology, Wuhan, China \\ Correspondence to: Daoyu Hu. No. 1095 Jiefang Avenue, Wuhan 430030, China. Email: daoyuhu@hust.edu.cn.
}

\begin{abstract}
Background: This study sought to explore the functional relationship between displayed vascular length and blood suppression inversion time (BSP TI) and flow velocity in a phantom, and to provide a theoretical basis for quantitatively assessing vascular hemodynamic responses using unenhanced magnetic resonance angiography (MRA) and spatial labeling with multiple inversion pulses sequence (SLEEK).

Methods: A polyethylene catheter was laid in a long rectangular container filled with pork fat. The entrance of the catheter into the container was connected to a high-pressure syringe filled with normal saline. The high-pressure injector flow rates were set at $0.0,0.2,0.4,0.8,1.2,1.6,2.0$, and $2.4 \mathrm{~mL} / \mathrm{s}$. SLEEK was performed 19 times for each flow rate with parameter BSP TI values of 50, 75, 100, 150, 200, 300, 400, 500, $600,700,800,900,1,000,1,100,1,200,1,300,1,400,1,600$, and 1,800 ms. Maximum intensity projection was employed to reconstruct all SLEEK original images to determine the measurements of the displayed vascular lengths. A regression analysis was undertaken to assess the relationship between the displayed vascular lengths and BSP TI values for each flow rate, and to assess the relationship between the displayed vascular lengths and flow rates at each BSP TI.
\end{abstract}

Results: The displayed vascular length had a linear relationship with BSP TI for each flow rate $(\mathrm{P}<0.05)$ $(\mathrm{R} 2=0.754,0.941,0.988,0.988,0.977,0.966$, and 0.982 for flow rates of $0.0,0.2,0.4,0.8,1.2,1.6$, and $2.0 \mathrm{~mL} / \mathrm{s}$, respectively). The displayed vascular length also had a linear relationship with flow rate for each BSP TI value $(\mathrm{P}<0.05)(\mathrm{R} 2=0.914,0.912,0.834,0.989,0.980,0.996,0.992,0.960,0.975,0.979,0.982,0.981$, 0.976, and 0.993 for BSP TI 50, 75, 100, 150, 200, 300, 400, 500, 600, 700, 800, 900, 1,000, and 1,100 ms, respectively). No significant linear relationship was found between displayed vascular length and flow rate when the BSP TI value was 1,200 $\mathrm{ms}(\mathrm{P}>0.05)$.

Conclusions: Vascular displayed length has a linear relationship to BSP TI for flow ranges from 0.0 to $2.0 \mathrm{~mL} / \mathrm{s}$. Vascular displayed length has a linear relationship to flow rate for BSP TI values of 50 to 1,100 ms. Flow rate can be assessed in relation to vascular displayed length.

Keywords: Unenhanced MR angiography; multiple inversion pulses; blood suppression inversion time; blood flow; vascular length; phantom

Submitted May 03, 2020. Accepted for publication Nov 24, 2020.

doi: 10.21037/qims-20-633

View this article at: http://dx.doi.org/10.21037/qims-20-633

\section{Introduction}

A variety of diseases can cause changes in the hemodynamics of abdominal blood vessels, leading to impaired target organ function; however, the vascular morphology does not change significantly. Non-invasive vascular morphology imaging and the quantitative analysis of hemodynamics can aid considerably in the early diagnosis and treatment of diseases. Currently, the main methods for the non-invasive 
measurement of blood flow velocity are color Doppler flow imaging (CDFI) and cine phase contrast (cine PC) in magnetic resonance technology (1). However, CDFI can easily be affected by a variety factors, such as obesity, intestinal gas, and examiners' experience, and is limited by the angle of the sound field, which makes it difficult to measure the blood flow perpendicular to the sound beam. Due to breathing movement and limited breath holding time, cine PC is mostly used to measure blood flow velocity and blood flow at a single plane for abdominal vessels (2). It is difficult for CDFI and cine PC to both clearly and completely display the vascular morphology at the same time.

Recently, the use of unenhanced MR angiography of balanced steady-state free precession sequences combined with arterial spin labeling (ASL) has attracted attention. In this method, a steady-state signal is largely determined by the T2:T1 ratio that is relatively high for blood. With the improvement of the hardware level, a short TR of less than 3-4 ms and a large flip angle are allowed, and the signal-tonoise ratio is greatly improved. Thus, parallel acquisition can be adopted to further speed up scanning $(3,4)$. However, in a balanced steady-state free precession sequence, the veins and arteries have high signals that cannot be distinguished. Thus, prepulse is used to selectively display the artery or vein $(5,6)$.

New unenhanced magnetic resonance angiography (MRA) technologies have been introduced, including time spatial labeling inversion pulse (Time-SLIP, Toshiba), which is a technology based on ASL (7), Native trueFISP (Siemens) (8), b-TRANCE (Philips), inflow inversion recovery (IFIR, GE) (9), and spatial labeling with multiple inversion pulses (SLEEK, GE Healthcare). SLEEK is an accurate method and has excellent concordance with digital subtraction angiography (DSA) in depicting the anatomy of the vessel of transplant renal and renal vessel $(10,11)$. The adoption of the optimal blood suppression inversion time (BSP TI) based on breath rate (BR) and heart rate (HR) can improve renal arterial visibility (12).

Takei et al. used inflow inversion recovery with fast spin echo to display carotid arteries in 10 healthy volunteers. The BSP TI value were 800, 1,200, 1,600, and 2,000 ms. As the BSP TI values increased, the length of the carotid artery increased (13). Previous studies have performed vascular morphological research of abdominal vessels. The present study used an extracorporeal vascular phantom to explore the relationship between BSP TI, fluid flow rate, and simulated vessel display length to evaluate the ability of unenhanced MRA with SLEEK technique in quantify fluid flow and clarify the flow direction.

\section{Methods}

\section{Phantom construction}

A small hole with a diameter of about $4 \mathrm{~mm}$ was made on each of the short sides of a long rectangular plastic container $(30 \mathrm{~cm} \times 8.5 \mathrm{~cm} \times 7.6 \mathrm{~cm}$ in size). The small holes were used to simulate blood vessels entering and exiting the container. A plastic tube with an inner diameter of $3 \mathrm{~mm}$ was passed through the small holes on both sides, and was run parallel to the long axis of the container (see Figure 1) to avoid any speed change caused by folding. A plastic bracket was then placed in the container to fix the plastic tube that was kept in the same horizontal plane throughout.

Melted lard was used for packing. The thickness of the lard on the horizontal surface of the plastic tube was more than $1 \mathrm{~cm}$, and the total thickness was approximately $3 \mathrm{~cm}$. Lard was used to simulate the fat around blood vessels in the body. The inlet of the plastic tube was connected to a high-pressure syringe (Stellant D, Medrad Inc., Warrendale, PA, USA), and the outlet was directed to a waste cup.

\section{SLEEK scan}

The long axis of the container was placed parallel to the $Z$ axis of the scanner and placed in the center of the scanning bed. Sponges were placed on the top and bottom of the container to secure the container and to ensure that the container was positioned in the center of the magnetic field. The high-pressure syringe, which contained physiological saline, was connected to a plastic tube, and all the tubes were filled with physiological saline to remove air bubbles.

All of the examinations were performed using a 1.5 T superconducting MR scanner (Signa Excite HD, GE Healthcare, Waukesha, WI, USA) with an 8-channel phased array cardiac coil. A three-plane positioning scan and a calibration scan were performed on the container, and then a fast imaging steady-state acquisition (FIESTA) sequence of coronal and axial positions was used for further positioning.

The flow rates of the high-pressure syringe were set at $0.0,0.2,0.4,0.8,1.2,1.6,2.0$ and $2.4 \mathrm{~mL} / \mathrm{s}$, that was $0,2.8$, $5.7,11.3,17.0,22.6,28.3$ and $34.0 \mathrm{~cm} / \mathrm{s}$. A SLEEK sequence of coronal position was performed at each flow rate. The parameters were as follows: slice thickness $=2 \mathrm{~mm}$, space $=$ 


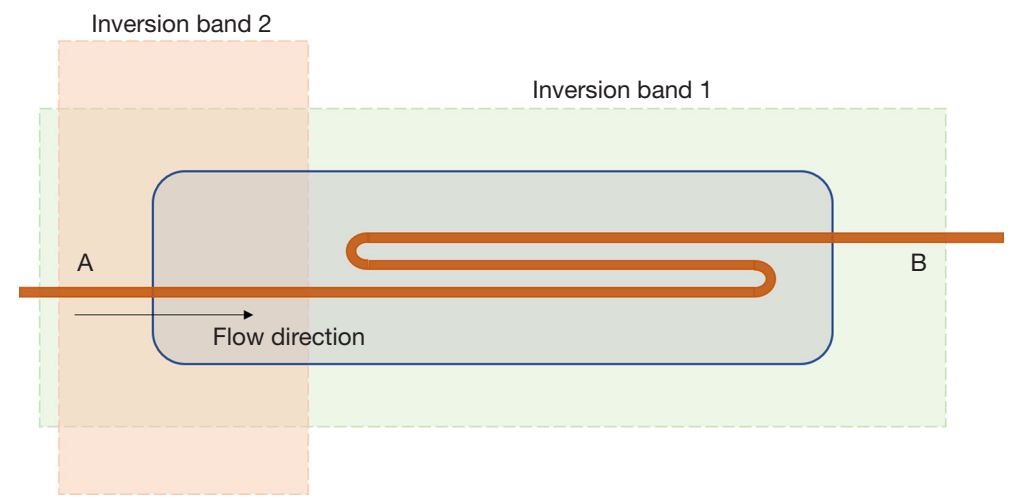

Figure 1 The rounded rectangle is the container, the green dashed rectangle and the pink dashed rectangle are the inverted bands, and the red line is the plastic tube. The plastic was is connected to a high-pressure syringe used to inject physiological saline, which flowed out from the $\mathrm{B}$ end. Inversion band 1 covered the entire container, and inverted physiological saline and background tissue signals. Inversion band 2 was placed above the turning point of the plastic tube at the A end (the entrance), and the signal of physiological saline and background tissue was reversed again, so that when the liquid in the tube of this section reached the downstream, it was collected by SLEEK as a high signal.

$1 \mathrm{~mm}, \mathrm{TE}=2.0 \mathrm{~ms}, \mathrm{TR}=3.9 \mathrm{~ms}$, flip angle $=75^{\circ}$, bandwidth $= \pm 125 \mathrm{kHz}$, field of view $(\mathrm{FOV})=40 \mathrm{~cm} \times 40 \mathrm{~cm}$, matrix $=224 \times 256$, respiratory interval $=1, \mathrm{NEX}=1$, sense factor $=2$, number of layers $=20$, and the inversion bands were placed in the same position. The scans were then performed 19 times under each flow rate, and the BSP TI values were set to $50,75,100,150,200,300,400,500,600,700,800$, $900,1,000,1,100,1,200,1,300,1,400,1,600$, and 1,800 ms. The assistant lay flat on the bed in the scanning room. Respiratory gating was tied to the assistant's abdomen, where breathing was most obvious. The assistant was asked to breathe smoothly, and maintain a breathing rate of about 18 breaths per minute. A single SLEEK scan took approximately 50 seconds. The scans were performed over 2 separate days, and each time a flow rate of $0 \mathrm{~mL} / \mathrm{s}$ was set.

SLEEK is a type of ASL technology. Two inversion bands that partially overlapped in the space were used. One inversion band was placed parallel to the long axis of the model container, covering all background tissues and blood, and reversing the macroscopic magnetization vector to M0. The other inversion band was placed in the simulated blood vessel entrance section. The blood at the entrance section that had been reversed to $-\mathrm{M} 0$ was reversed to $+\mathrm{M} 0$ again, and was marked. After a certain period (BSP TI), the labeled blood in this part flowed into the target area. At this time, a signal was collected from the target area to display the simulated blood vessel. For an explanation of the principle of SLEEK sequence technology, see Tang et al. (14). Figure 1 shows the placement of the two inversion bands in the present experiment. Notably, the high-pressure syringe was located outside the two inversion bands.

\section{Image analysis}

All of the original data from SLEEK were transmitted to an imaging workstation (Advantage Workstation 4.4, GE Healthcare, Buc, France) for processing. Maximum intensity projection with a thickness of $15 \mathrm{~mm}$ was used to display the simulated blood vessel. The length of the displayed simulated blood vessel was measured using maximum intensity projection. The starting point of the measurement was the location where the plastic tube entered the container, and the end point was the farthest point of the high signal in the plastic tube.

\section{Statistical analysis}

Statistical analysis was performed using SPSS 20 software (IBM, Armonk, NY, USA). The lengths of the displayed simulated blood vessel at different rates are expressed as $\bar{x} \pm s$. When the blood flow velocity was $0.0 \mathrm{~mL} / \mathrm{s}$, an independent samples $t$-test was used. For each fluid flow rate, the relationship between the length of the simulated vessel and the BSP TI value was evaluated using a single-factor linear regression analysis. For each BSP TI value, the relationship between the length of the simulated blood vessel and the fluid flow rate was evaluated using a single-factor linear regression analysis. 
Table 1 Relationship between the simulated blood vessel length and the BSP TI value

\begin{tabular}{lccccc}
\hline Flow rate $(\mathrm{mL} / \mathrm{s})$ & B1 value & Constant & $\mathrm{F}$ & $\mathrm{p}$ & $\mathrm{R}^{2}$ \\
\hline 0.0 & 0.029 & 62.846 & 82.662 & 0.000 & 0.754 \\
0.2 & 0.103 & 54.743 & 256.985 & 0.000 & 0.941 \\
0.4 & 0.191 & 66.212 & 929.576 & 0.000 & 0.988 \\
0.8 & 0.237 & 78.063 & $1,245.438$ & 0.000 & 0.988 \\
1.2 & 0.355 & 80.880 & 501.888 & 0.000 & 0.977 \\
1.6 & 0.410 & 98.953 & 229.152 & 0.000 & 0.966 \\
2.0 & 0.482 & 108.36 & 378.724 & 0.000 & 0.982 \\
\hline
\end{tabular}

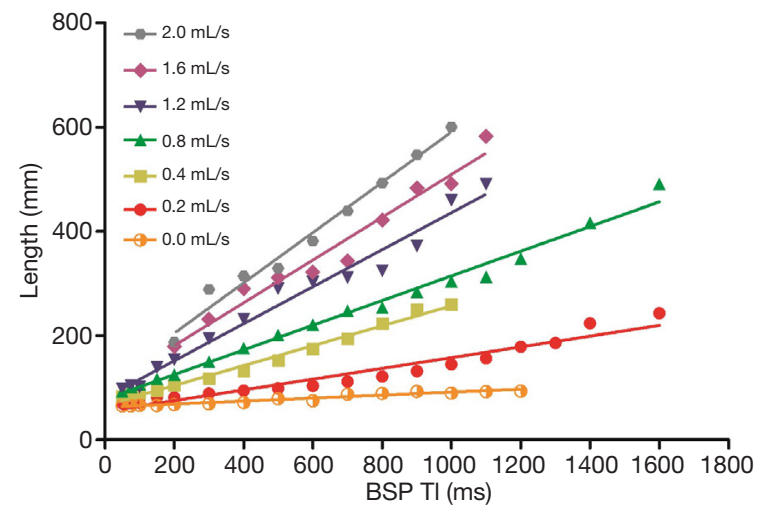

Figure 2 The relationship between simulated blood vessel length and BSP TI. The linear relationship between simulated blood vessel length and BSP TI value at each fluid flow rate.

\section{Results}

When the fluid flow rate was $0.0 \mathrm{~mL} / \mathrm{s}$, the simulated blood vessel length in the first scan was $82.09 \pm 13 \mathrm{~mm}$, and the simulated blood vessel length in the second scan was $73.69 \pm 11 \mathrm{~mm}$. The $t$ value was $1.897(\mathrm{P}=0.069)$. Thus, the difference between the two lengths at different days was not statistically significant. At each liquid flow rate, there was a linear relationship between the simulated blood vessel length and the BSP TI value $(\mathrm{P}<0.05)$. The larger the liquid flow rate, the larger the slope (see Table 1, and Figures 2,3). For the BSP TI values of 50, 75, 100, 150, 200, $300,400,500,600,700,800,900,1,000$, and $1,100 \mathrm{~ms}$, a linear relationship was found between simulated blood vessel length and fluid flow rate $(\mathrm{P}<0.05)$. However, no such linear relationship was found for the BSP TI value of $1,200 \mathrm{~ms}$ $(\mathrm{P}>0.05)$ (see Table 2, and Figures 4,5).

Contour maps were drawn of BSP TI (ms, horizontal axis), fluid flow (mL/s, vertical axis), and simulated vessel length $(\mathrm{mm} / \mathrm{s})$ (see Figure 6). Different colors areas in the picture represent different lengths. At higher fluid flow rates, shorter BSP TI values were required to display simulated blood vessels of the same length. When the fluid flow rate was $0.8 \mathrm{~mL} / \mathrm{s}$, the simulated intravascular fluid velocity was $11.3 \mathrm{~cm} / \mathrm{s}$. When the length to be displayed was $263-331 \mathrm{~mm}$, the applicable BSP TI range was 900 to $1,200 \mathrm{~ms}$. When the fluid volume was $1.2 \mathrm{~mL} / \mathrm{s}$, the simulated intravascular fluid velocity was $17.0 \mathrm{~cm} / \mathrm{s}$, and when the length to be displayed was $263-331 \mathrm{~mm}$, the applicable BSP TI values ranged from 500 to $800 \mathrm{~ms}$.

\section{Discussion}

SLEEK technology is a type of ASL that uses two inversion bands: one reverses all background signals, and the other is placed at the entrance region of the target vessel, and the blood signal within it is reversed and marked. The marked blood flow fills the distal blood vessels, and thus was visualized. In theory, blood flow velocity can be calculated (15) if the distance between the labeled blood and the signal acquisition area, and the period between when the blood is labeled and when the image is acquired, are known.

In the present study, a blood vessel phantom was used. A fluid flow rate (ranging from 0 to $2.0 \mathrm{~mL} / \mathrm{s}$ ) was set for the same BSP TI value, and the length of the displayed simulated blood vessel was then measured. A linear relationship was found between the length of the simulated blood vessel and the fluid flow $(\mathrm{P}<0.05)$, indicating that the greater the blood flow velocity was, the greater the length of the blood vessel displayed.

The fluid flow in the simulated blood vessel was set, the BSP TI was changed (from 50, 75, 100, 150, 200, 300, 


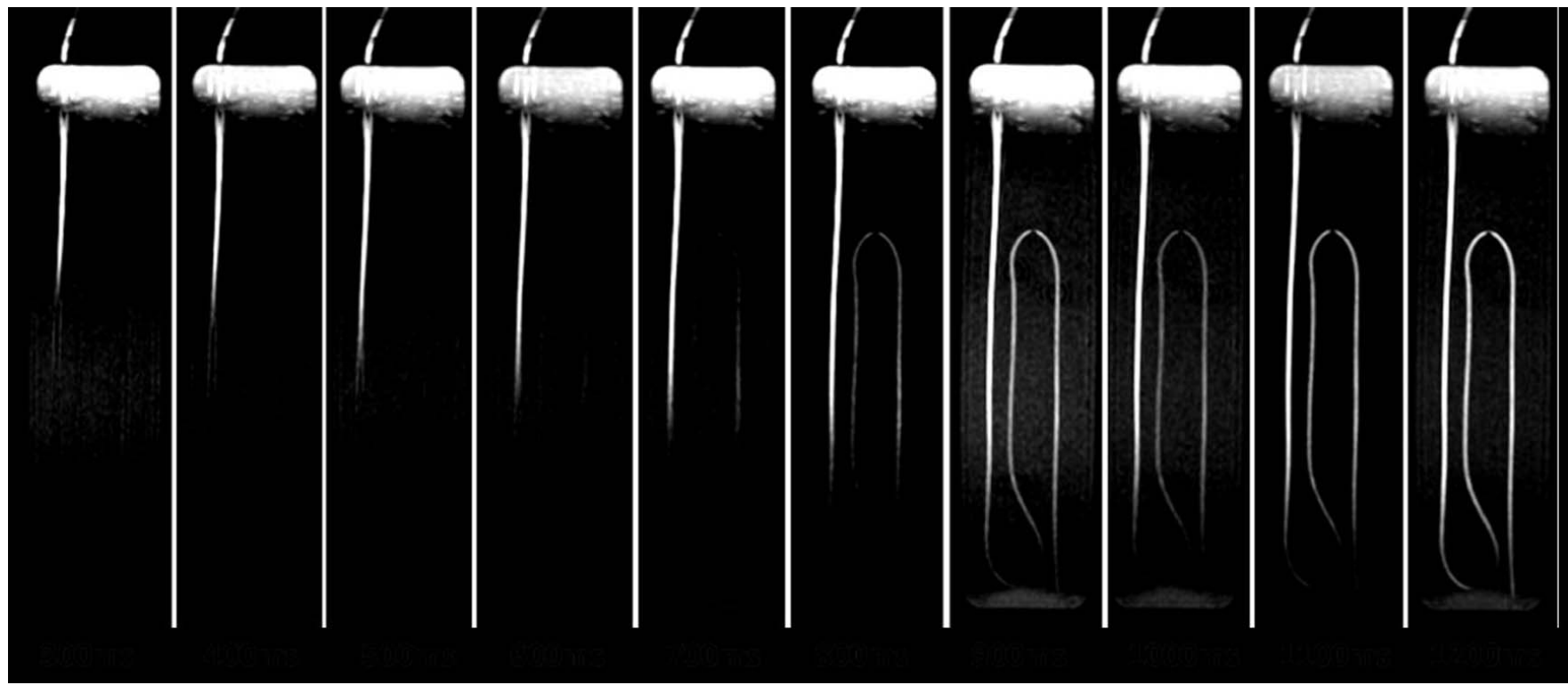

Figure 3 The white line is the high signal of the normal saline in the plastic tube, and the plastic tube inlet appears above. The highpressure syringe was set at the same flow rate $(0.4 \mathrm{~mL} / \mathrm{s})$, and the BSP TI values were 300, 400, 500, 600, 700, 800, 900, 1,000, 1,100, and $1,200 \mathrm{~ms}$. With the increase of BSP TI, the lengths of the simulated blood vessels gradually increased. When the BSP TI value was greater than $1,100 \mathrm{~ms}$, the farthest point of the high signal exceeded the first turn.

Table 2 Relationship between the simulated blood vessel length and fluid flow rate at each BSP TI value

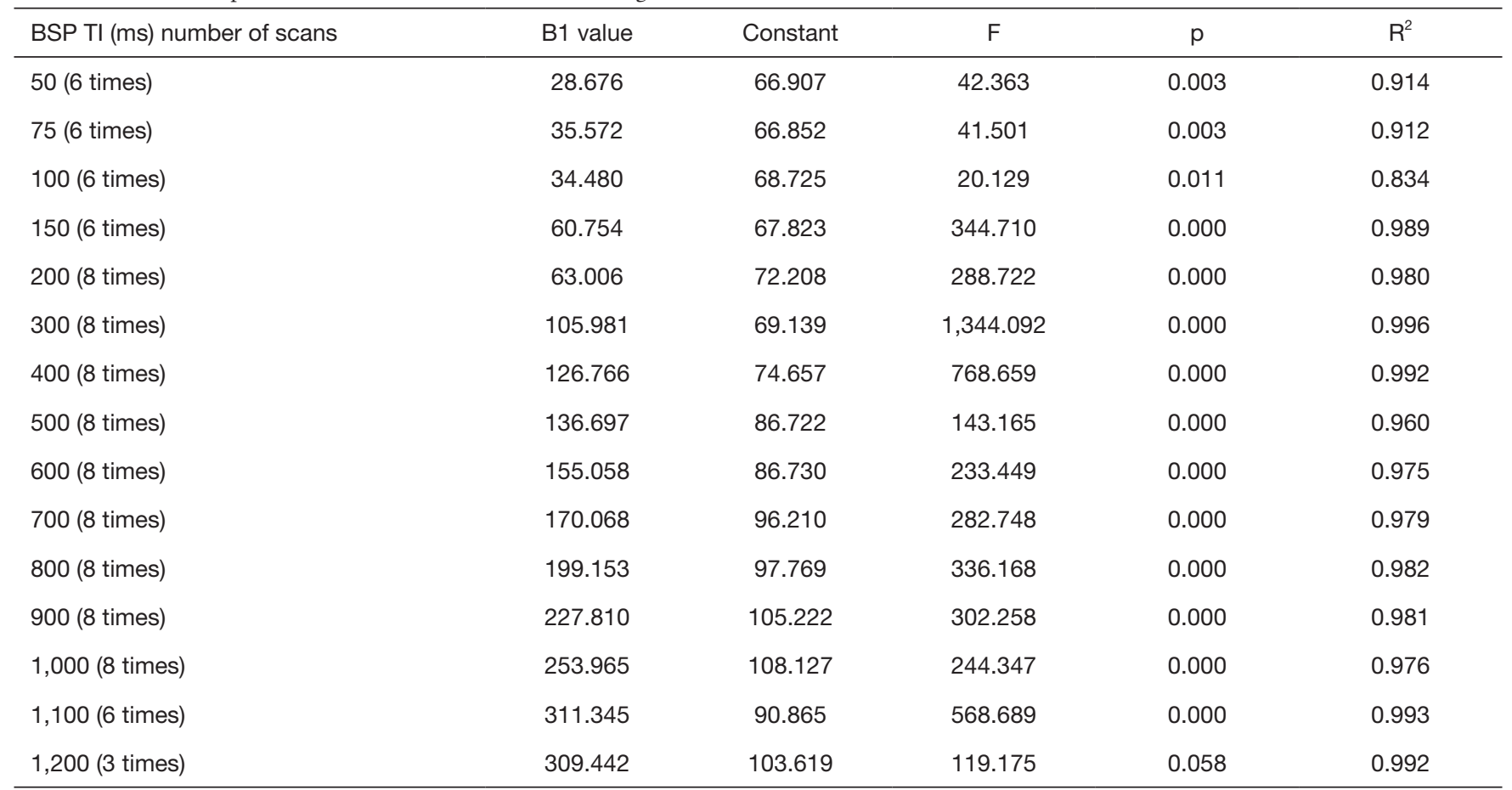


$400,500,600,700,800,900,1,000$, to $1,100 \mathrm{~ms})$, and the displayed simulated blood vessel length was measured. The results showed that there was a linear relationship between the simulated blood vessel length and the BSP TI value $(\mathrm{P}<0.05)$, and that the longer the BSP TI was, the longer

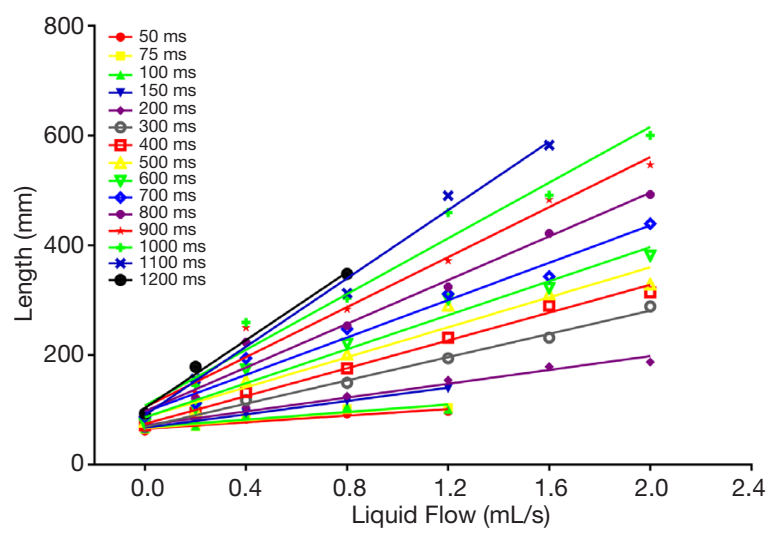

Figure 4 Simulated blood vessel length changed with fluid flow. When the BSP TI values were 50, 75, 100, 150, 200, 300, 400, 500, $600,700,800,900,1,000$, and 1,100 ms, the simulated blood vessel length had a linear relationship with the fluid flow rate. the length of the blood vessel displayed. If blood flow velocity is kept constant, longer blood vessels can be imaged by choosing a longer BSP TI value. To display simulated blood vessels of the same length, a smaller BSP TI value can be chosen when blood flow velocity is high.

Contour maps of the flow rates, BSP TI values, and the simulated blood vessel lengths were plotted. The results showed that at certain simulated blood vessel lengths, if a smaller BSP TI value was used to display the full length, the flow was large. Time delay affects signal strength because as T1 relaxation recovers, longer time delays can decrease differences between inverted and uninverted signals (16). In the present study, we noted that as the BSP TI values increased, the signal of the liquid that had been reversed only once gradually increased, especially in a scan with a flow rate of $0.0 \mathrm{~mL} / \mathrm{s}$. When the BSP TI value was $900 \mathrm{~ms}$, the signal of the liquid that had been reversed only once started to show a relatively uniform slightly high signal over the entire section. When the BSPTI was higher than $1,400 \mathrm{~ms}$, the signal intensity increased further, and it was difficult to distinguish it from the marked liquid that had been inverted twice.

For renal vascular imaging, it is necessary to add

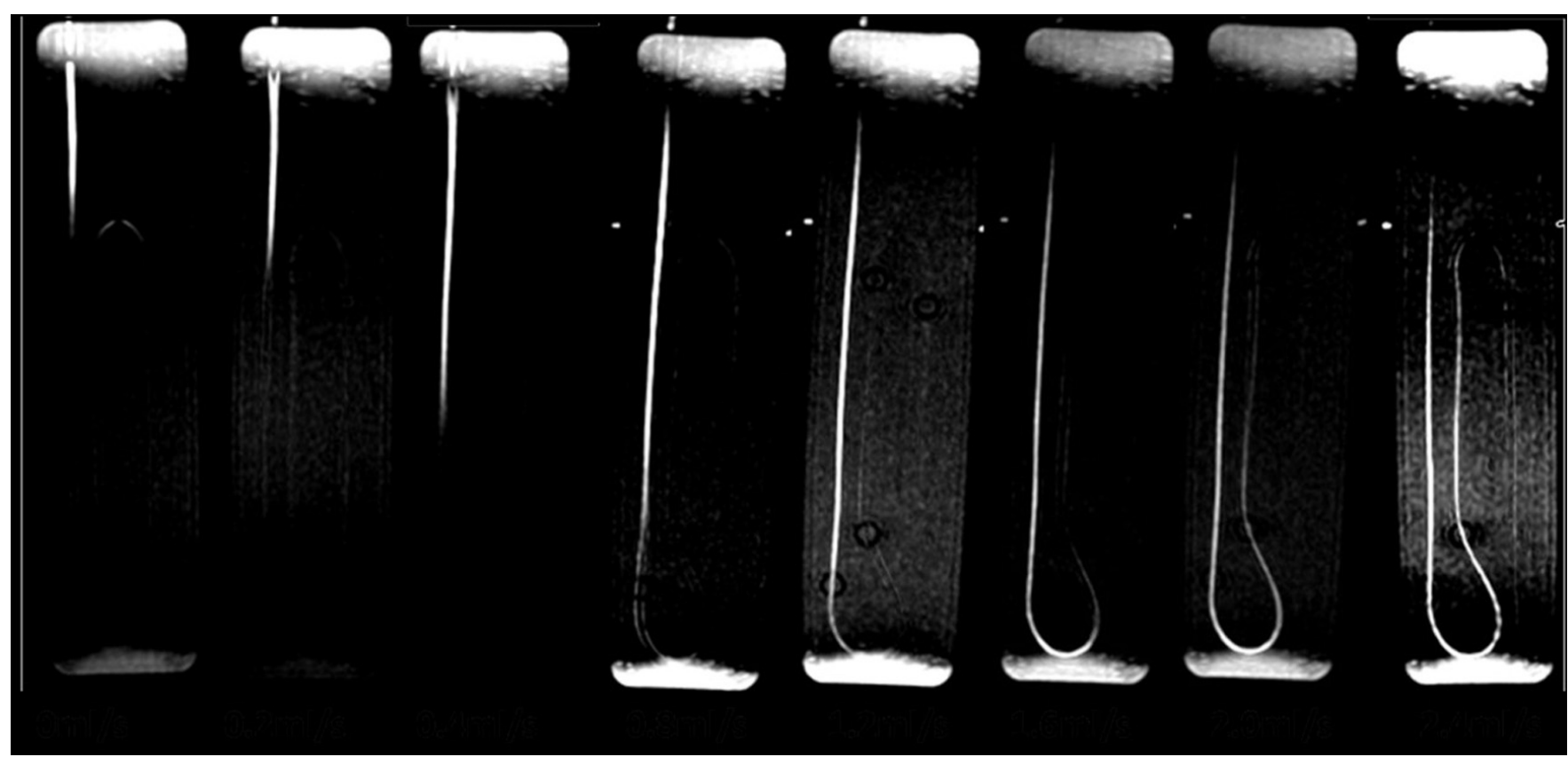

Figure 5 The white line is the high signal of normal saline in the plastic tube, and the plastic tube inlet appears above. The BSP TI was set to $600 \mathrm{~ms}$, and the liquid flow rates of the high-pressure syringe were $0,0.2,0.4,0.8,1.2,1.6,2.0$, and $2.4 \mathrm{~mL} / \mathrm{s}$. With the increase of flow rate, the lengths of the simulated blood vessels gradually increased. When the liquid flow was greater than 1,100 ms, the farthest point of the high signal exceeded the first turn. 


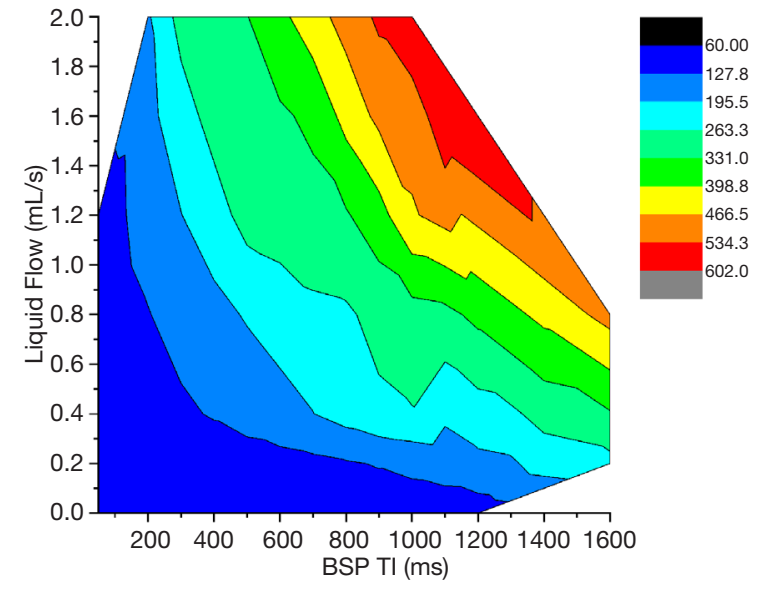

Figure 6 Contour plot of BSP TI value, fluid flow rate, and simulated vessel length.

saturation bands in the renal area and inferior vena cava to reduce venous signals, while simultaneously applying fat suppression to reduce fat interference (17). In the present study, the fat signal changed as the BSP TI values changed. Specifically, the fat signal was higher for smaller BSP TI values, but decreased with the increase of BSP TI values, before slowly rising again. However, this had no effect on the measurements of the simulated blood vessel lengths.

In the present study, we observed that as BSP TI values increased, signal dilution, thinning, doubling , or bifurcation appeared at the far end of the simulated angiography, and the boundary with the unlabeled fluid became blurred. There are several possible reasons for these results. First, this might have been due to Brownian motion and the magnetization of liquid molecules. Second, perhaps when the flow velocity is small, the liquids is laminar, and the degree of confusion is light, and conversely, when the flow velocity is large, the vortices are generated in the duct. Third, it is possible that while a large arc was used in the bend of the pipe to ensure a smooth pipe diameter, the plastic pipe at the bend flattened, as the pipe was flexible, and the complicated fluid direction at the bends and far section resulted in phase loss and signal loss. This would be consistent with previous studies that have reported that this type of technique exaggerates the degree of stenosis due to the poor visualization of the distal stenosis in human renal artery stenosis (18).

In conclusion, the present study identified the relationships between BSP TI and displayed simulated vessel length, and fluid flow and simulated vessel length.
If two of the three parameters are known, the range of the other parameter can be roughly inferred. This may provide a theoretical basis for using SLEEK to perform non-invasive hemodynamic assessments and angiographic morphological assessments simultaneously.

\section{Acknowledgments}

Funding: National Natural Science Foundation of China (NSFC); contract grant number: 81571642, 81771801, 81701657.

\section{Footnote}

Conflicts of Interest: All authors have completed the ICMJE uniform disclosure form (available at http://dx.doi. org/10.21037/qims-20-633). The authors have no conflicts of interest to declare.

Open Access Statement: This is an Open Access article distributed in accordance with the Creative Commons Attribution-NonCommercial-NoDerivs 4.0 International License (CC BY-NC-ND 4.0), which permits the noncommercial replication and distribution of the article with the strict proviso that no changes or edits are made and the original work is properly cited (including links to both the formal publication through the relevant DOI and the license). See: https://creativecommons.org/licenses/by-nc-nd/4.0/.

\section{References}

1. Long J, Lin H, Cao G, Wang M, Huang X, Xia J, Sun Z. Relationship between intracranial pressure and phasecontrast cine MRI-derived measures of cerebrospinal fluid parameters in communicating hydrocephalus. Quant Imaging Med Surg 2019;9:1413-20.

2. Kadoya Y, Miyati T, Kobayashi S, Ohno N, Gabata T. Evaluation of gravity effect on inferior vena cava and abdominal aortic flow using multi-posture MRI. Acta Radiol 2020. [Epub ahead of print]. doi: 10.1177/0284185120950112.

3. Shimada K, Isoda H, Okada T, Kamae T, Arizono S, Hirokawa Y, Shibata T, Togashi K. Non-contrast-enhanced hepatic MR angiography: Do two-dimensional parallel imaging and short tau inversion recovery methods shorten acquisition time without image quality deterioration? Eur J Radiol 2011;77:137-42.

4. Xu J, McGorty KA, Lim RP, Bruno M, Babb JS, 
Srichai MB, Kim D, Sodickson DK. Single breathhold noncontrast thoracic MRA using highly accelerated parallel imaging with a 32-element coil array. J Magn Reson Imaging 2012;35:963-8.

5. Ishimori $Y$, Monma $M$, Kawamura $H$, Miyata $T$. Time spatial labeling inversion pulse cerebral MR angiography without subtraction by use of dual inversion recovery background suppression. Radiol Phys Technol 2011;4:78-83.

6. Okuaki T, Ishimoto T, Miyati T, Kobayashi S, Ishihara M, Kawakami M, Ogino T, Van Cauteren M. Separate pulmonary artery and vein magnetic resonance angiography by use of an arterial spin labeling method. Radiol Phys Technol 2014;7:352-7.

7. Hamamoto K, Matsuura K, Chiba E, Okochi T, Tanno K, Tanaka O. Feasibility of Non-contrast-enhanced MR Angiography Using the Time-SLIP Technique for the Assessment of Pulmonary Arteriovenous Malformation. Magn Reson Med Sci 2016;15:253-65.

8. Değirmenci B, Kara M, Kıdır V, İnal S, Sezer T, Umul A, Orhan H, Çelik AO, Demirtaş H, Yilmaz Ö. Unenhanced respiratory-navigated NATIVE ® TrueFISP magnetic resonance angiography in the evaluation of renal arteries: Comparison with contrast-enhanced magnetic resonance angiography. Diagn Interv Imaging 2017;98:133-40.

9. Xu X, Lin X, Huang J, Pan Z, Zhu X, Chen K, Zee C, Yan $\mathrm{F}$. The capability of inflow inversion recovery magnetic resonance compared to contrast-enhanced magnetic resonance in renal artery angiography. Abdom Radiol (NY) 2017;42:2479-87.

10. Tang H, Wang Z, Wang L, Hu X, Wang Q, Li Z, Li J, Meng X, Wang Y, Hu D. Depiction of Transplant Renal Vascular Anatomy and Complications: Unenhanced MR Angiography by Using Spatial Labeling with Multiple Inversion Pulses. Radiology 2014;271:879-87.

11. Pei Y, Shen H, Li J, Zhang H, Xia L, Wang L, Hu D.

Cite this article as: Chen X, Meng X, Zhu D, Zou X, Shen Y, Li Z, Peng J, Hu D. Hemodynamic study of unenhanced magnetic resonance angiography using spatial labeling with multiple inversion pulses sequence: a phantom study. Quant Imaging Med Surg 2021;11(5):1828-1835. doi: 10.21037/qims20-633
Evaluation of Renal Artery in Hypertensive Patients by Unenhanced MR Angiography Using Spatial Labeling With Multiple Inversion Pulses Sequence and by CT Angiography. AJR Am J Roentgenol 2012;199:1142-8.

12. Pei Y, Li F, Shen H, Long X, Liu H, Wang X, Liu J, Li W. Optimal Blood Suppression Inversion Time Based on Breathing Rates and Heart Rates to Improve Renal Artery Visibility in Spatial Labeling with Multiple Inversion Pulses: A Preliminary Study. Korean J Radiol 2016;17:69.

13. Takei N, Miyoshi M, Kabasawa H. Noncontrast MR angiography for supraaortic arteries using inflow enhanced inversion recovery fast spin echo imaging. J Magn Reson Imaging 2012;35:957-62.

14. Tang H, Wang Z, Wang L, Hu X, Wang Q, Li Z, Li J, Meng X, Wang Y, Hu D. Depiction of transplant renal vascular anatomy and complications: unenhanced $M R$ angiography by using spatial labeling with multiple inversion pulses. Radiology 2014;271:879-87.

15. Barbier EL, Silva AC, Kim SG, Koretsky AP. Perfusion imaging using dynamic arterial spin labeling (DASL). Magn Reson Med 2001;45:1021-9.

16. Williams DS. Quantitative perfusion imaging using arterial spin labeling. Methods Mol Med 2006;124:151-73.

17. Coenegrachts KL, Hoogeveen RM, Vaninbroukx JA, Bosmans HT, Bielen DJ, Maleux G, Maes F, Hamaekers P, Oyen RH, Marchal GJ. High-Spatial-Resolution 3D Balanced Turbo Field-Echo Technique for MR Angiography of the Renal Arteries: Initial Experience. Radiology 2004;231:237-42.

18. Wyttenbach R, Braghetti A, Wyss M, Alerci M, Briner L, Santini P, Cozzi L, Di Valentino M, Katoh M, Marone C, Vock P, Gallino A. Renal Artery Assessment with Nonenhanced Steady-State Free Precession versus Contrast-enhanced MR Angiography. Radiology 2007;245:186-95. 